\title{
Genetic diversity and population differentiation in the cockle Cerastoderma edule estimated by microsatellite markers
}

\author{
L. Martínez $\cdot$ J. Méndez $\cdot$ A. Insua $\cdot$ \\ A. Arias-Pérez $\cdot$ R. Freire
}

Received: 3 October 2011/Revised: 21 May 2012 / Accepted: 9 June 2012/Published online: 23 June 2012

(C) Springer-Verlag and AWI 2012

\begin{abstract}
The edible cockle Cerastoderma edule is a marine bivalve commercially fished in several European countries that have lately suffered a significant decrease in production. Despite its commercial importance, genetic studies in this species are scarce. In this work, genetic diversity and population differentiation of $C$. edule has been assessed using 11 microsatellite markers in eight locations from the European Atlantic coast. All localities showed similar observed and expected heterozygosity values, but displayed differences in allelic richness, with lowest values obtained for localities situated farther north. Global $F$ st value revealed the existence of significant genetic structure; all but one locality from the Iberian Peninsula were genetically homogeneous, while more remote localities from France, The Netherlands, and Scotland were significantly different from all other localities. A combined effect of isolation by distance and the existence of barriers that limit gene flow may explain the differentiation observed.
\end{abstract}

Keywords Microsatellite markers - Cerastoderma . Genetic variability $\cdot$ Population structure

Communicated by Heinz-Dieter Franke.

L. Martínez · J. Méndez · A. Insua · A. Arias-Pérez .

R. Freire $(\bowtie)$

Departamento de Biología Celular y Molecular,

Área de Genética, Facultad de Ciencias,

Universidade da Coruña, A Zapateira s/n,

15071 A Coruña, Spain

e-mail: rfreire@udc.es

\section{Introduction}

Many bivalve fisheries are currently being overexploited due to the increasing demand for seafood on a global scale. To avoid compromising the survival of these fisheries, management strategies comprising a better understanding of bivalve biology are necessary. In particular, the characterization of genetic diversity and a good understanding of population structure are important for delineating management units and maintaining the evolutionary potential. Even though low or no genetic structure is expected for most marine organisms, given their high dispersal potential and the absence of evident barriers in most of the marine environment, there are increasing reports of differentiation of bivalve populations over large and small scales (Reeb and Avise 1990; Ridgway 2001; Luttikhuizen et al. 2003; Arias et al. 2011). Exploitation without taking into account the genetic background can cause alterations of the genetic subdivision of populations and loss of genetic variation (Allendorf et al. 2008), decreasing their fitness and adaptive potential.

The cockle Cerastoderma edule (Bivalvia: Cardiidae) is a filter-feeding bivalve that inhabits the marine soft-bottom intertidal and shallow subtidal along the European coast. Its distribution comprises the eastern Atlantic coast, from Senegal in western Africa to the Barents Sea, reaching into marginal seas like the Baltic and the Mediterranean (Hayward and Ryland 1995). Cockles are relatively short lived, mature quickly, and have a high fecundity (Honkoop and van der Meer 1998) with very variable recruitment and population size. Cockle larvae are exposed to tidal currents and drift for approximately 30 days before metamorphosing and settling to the seabed as postlarvae (Dare et al. 2004). Cerastoderma edule plays a key role in coastal food webs of the northeast Atlantic and is commercially fished 
in several countries including United Kingdom, Spain, France, and Portugal. According to FAO statistics, European production of this species has suffered a sharp decrease, going from 107,794 tons in 1987 to 24,799 tons in 2008. Its overexploitation is especially evident in The Netherlands, being the main producing country during the 80 and 90s, with a maximum of 76,349 tons in 1989 , but with no registered production nowadays.

Despite its commercial importance, few studies regarding population genetics in $C$. edule have been carried out. Most of them are based on allozyme markers. Beaumont et al. (1980) analyzed genetic variation at the Octopine dehydrogenase (Odh) locus in four localities coming from Wales, France, and The Netherlands, and they found significant differences between localities situated east and west of the English Channel. By contrast, Hummel et al. (1994) studied seven allozyme loci and found high gene flow among localities from Denmark, The Netherlands, and France. Beaumont and Pether (1996), by means of nine loci, concluded that there was little differentiation of cockle beds around southern UK, without any clear separation between western and eastern localities. In a recent study, Krakau et al. (2012) used mitochondrial cytochrome oxidase subunit I (COI) to assess the genetic variability in 19 sampling sites. They found two dominant central haplotypes separated by low divergence.

This study aims to assess the genetic diversity and population differentiation of eight European localities using microsatellites as molecular markers. Being more polymorphic than allozymes and mitochondrial markers, microsatellites should be more powerful to detect genetic structure and outcrossing rates.

\section{Materials and methods}

Sample collection and DNA extraction

Samples of $C$. edule were collected from eight locations (Fig. 1): Aveiro (Portugal), San Simón, Boiro, Corcubión and Ferrol (northwest Spain), Brest (France), Oosterschelde (The Netherlands), and Isle of Lewis (Scotland). Total genomic DNA was extracted from the adductor muscle according to Fernández-Tajes and Méndez (2007). For samples collected from Spain, DNA extraction was carried out from fresh tissue, while for the rest of localities, cockle tissue was preserved in ethanol until extraction.

Genetic variation

Individual genotypes were determined for 11 polymorphic microsatellites according to Martínez et al. (2009). Allele frequencies, observed number of alleles per locus $\left(N_{\mathrm{A}}\right)$,

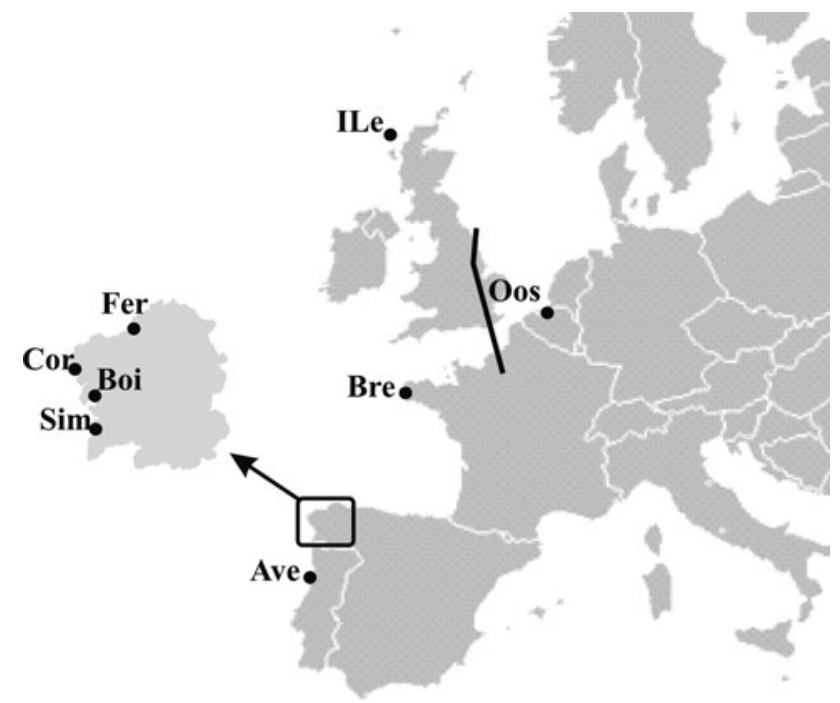

Fig. 1 Sampling sites location: Aveiro (Ave); San Simón (Sim); Boiro (Boi); Corcubión (Cor); Ferrol (Fer); Brest (Bre); Oosterschelde (Oos); Isle of Lewis (ILe). Continuous line indicates a potential barrier to gene flow as identified by Barrier software

observed heterozygosity $\left(H_{\mathrm{o}}\right)$, and unbiased expected heterozygosity $\left(H_{\mathrm{e}}\right)$ of Nei (1978) were obtained with the Genetix v.4.03 software (Belkhir et al. 2004). Allelic richness $(R \mathrm{~s})$ per locus and per locus-locality combination was computed with Fstat v. 2.9.3.2 (Goudet 2002). A Friedman test was carried out to compare allelic richness between localities with the statistical package SPSS 16.0 (SPSS Inc.). A post hoc analysis on the Friedman test was carried out as described in Bortz et al. (2000).

Tests for agreement with Hardy-Weinberg equilibrium (HWE) and linkage disequilibrium between pairs of loci within each locality were carried out with Genepop v.4.0 software (Rousset 2008). The significance was determined by a Markov chain method using 10,000 dememorizations, 1,000 batches, and 5,000 iterations per batch. MicroChecker software (Van Oosterhout et al. 2004) was used to examine microsatellite data for evidence of null alleles and their frequency was estimated following Brookfield (1996). The frequency of private alleles in each locality was tested for correlation with sample size. Should multiple tests be carried out, $P$ values were adjusted using the sequential Bonferroni correction (Rice 1989).

Both the Ewens-Watterson homozygosity test (Watterson 1978; Watterson 1986) and the Ewens-WattersonSlatkin exact test (Slatkin 1994; Slatkin 1996) were carried out to check for departures from selective neutrality using Arlequin 3.11 software (Excoffier et al. 2005).

Population differentiation

Tests for genic and genotypic differentiation for all pairs of localities were performed using Genepop v.4.0 software. 
Weir and Cockerham's $F$ statistics (1984) computed over all localities, over all loci, and on a pair-wise basis between localities were obtained using Genetix v.4.03 software, which was also used to determine probability of significance of $F$ st values by a nonparametric permutation approach $(10,000$ permutations). Since the $F$ st value is highly dependent on the level of genetic variation, making the interpretation and comparison of the level of genetic differentiation between loci and studies difficult, a standardized measure $\left(F\right.$ st'$\left.^{\prime}\right)$ was calculated in an analogous way to Hedrick's approach (2005). That is, the observed value of $F$ st was divided by the maximum level of genetic differentiation that can be obtained for the observed amount of genetic variation. This maximum value was calculated by recoding the data such that all populations only contain unique alleles. The standardized measure was interpreted as equal to zero when negative values of $F$ st were obtained. When multiple tests were performed, significance values were adjusted using the sequential Bonferroni correction.

A hierarchical analysis of molecular variance (AMOVA) locus by locus was conducted using Arlequin 3.11 software to assess the component of genetic diversity attributable to variance among groups (different regional groupings were tested), variance among localities within groups, and variance within localities.

A Mantel test with 10,000 randomizations, as implemented in software IBDWS v.3.16 (Jensen et al. 2005), was also carried out to check for linear correlation between genetic differentiation $(F \mathrm{st} /(1-F \mathrm{st}))$ and the natural $\log$ of geographical distance between sample pairs (measured as the coastline distance $(\mathrm{km})$ between sampling locations). Latitude and longitude coordinates were obtained for each sampling location and used along with the genetic data collected from the microsatellite analysis to generate a connectivity network of genetic distances based on Delaunay triangulation using Barrier v.2.2 software (Manni et al. 2004). Monmonier's maximum difference algorithm was then used to identify the main putative genetic boundary across the oceanographic landscapes. Significance was established by bootstrap (100 replicates used).

\section{Results}

Genetic variation statistics by locus, locality, and overall are shown in Table 1. All loci analyzed were polymorphic in all localities; number of alleles per locus ranging from eight (CeATC2-44) to 48 (CeATC2-11). Allelic richness across localities per locus varied between 5.052 (CeATC136) and 19.627 (CeATC1-5). Expected heterozygosity per locus ranged from 0.384 (CeATC1-36) to 0.929 (CeATC15 ), and observed heterozygosity from 0.229 (CeATC2-12) to 0.821 (CeATC1-22). For each locus-locality pair, allelic richness ranged from 4.235 (CeATC1-36 in Aveiro) to 21.348 (CeATC1-5 in Ferrol).

Regarding localities, Friedman test $(P=0.016)$ detected significant differences in allelic richness, and post hoc analysis showed that Isle of Lewis' allelic richness was significantly different from those of Ferrol and Brest ( $P=0.004$ and $P=0.037$, respectively). Sixty-three out of the 248 alleles detected across loci were private, although their frequency was always lower than 0.05 . The number of private alleles was strongly correlated with sample size $\left(F_{[1,6]}=22.992, P=0.003, R_{a d j}^{2}=0.793\right)$. All localities showed similar values of expected and observed heterozygosity, ranging from 0.729 (Corcubión) to 0.756 (Oosterschelde) and from 0.535 (Brest) to 0.602 (Boiro), respectively, and for all of them, values of expected heterozygosity were higher than those of observed heterozygosity.

Linkage disequilibrium tests performed for all pair of loci across localities gave no significant values $(P>0.05)$ for the 440 comparisons analyzed. Fifty-five out of the 88 locality-locus combinations showed no significant deviations from HWE after sequential Bonferroni correction. Four out of the 11 loci conformed to HWE in all localities (CeATC1-22, CeATC1-36, CeATC1-52, and CeATC2-34), while three (CeATC1-5, CeATC2-12, and CeATC2-51) showed significant departures from HWE in almost all localities. All combinations departing from HWE expectations but one showed positive $F$ is values, which indicates the existence of a heterozygote deficit. The exception comprises locus CeATC2-44 in Aveiro, which shows significant departure from HWE, although $F$ is value for the combination is very close to zero. This is due to the fact that even though global homozygote and heterozygote frequencies do not differ from expected values, distribution of heterozygote genotypes is not consistent with predicted values. Deviations from HWE were also detected for all localities across loci, all of which display positive $F$ is values ranging from 0.200 (Boiro) to 0.272 (Brest).

According to Microchecker software, eight loci showed evidence of the presence of null alleles, three (CeATC1-5, CeATC2-12, and CeATC2-51) at high frequencies $(>0.2)$ for several localities. Allele frequencies were corrected for putative null alleles using FreeNa software (Chapuis and Estoup 2007). Nevertheless, the estimated allele frequencies and the outcome of the statistical tests of differentiation were very similar to the original ones and only these are presented. Moreover, taking into account that the presence of null alleles at frequencies above 0.2 leads to a considerable overestimation of $F$ st estimators and genetic distance (Chapuis and Estoup 2007), these three loci were removed from subsequent analyses.

Ewens-Watterson homozygosity test reported deviations from neutrality at locus CeATC1-5 in Aveiro, San 
Table 1 Summary statistics of genetic diversity within C. edule

\begin{tabular}{|c|c|c|c|c|c|c|c|c|c|}
\hline & Ave & Sim & Boi & Cor & Fer & Bre & Oos & ILe & Total \\
\hline \multicolumn{10}{|c|}{ СeATC1-5 } \\
\hline$N$ & 57 & 49 & 45 & 40 & 56 & 39 & 73 & 38 & 397 \\
\hline$N_{A}$ & $22(1)$ & $21(1)$ & $19(1)$ & $21(0)$ & $26(5)$ & $22(2)$ & $20(1)$ & $12(0)$ & 36 \\
\hline$R$ & $195-261$ & $195-290$ & $184-258$ & $192-258$ & $171-380$ & $192-380$ & $192-397$ & $192-243$ & $171-397$ \\
\hline$R_{S}$ & 19.486 & 19.089 & 17.513 & 19.248 & 21.348 & 20.984 & 17.146 & 11.367 & 19.627 \\
\hline$A_{C}$ & 207 & 243 & 240 & 207 & 198 & 195 & 207 & 207 & 207 \\
\hline$A_{C}$ freq & 0.123 & 0.112 & 0.133 & 0.150 & 0.134 & 0.115 & 0.288 & 0.382 & \\
\hline$H_{e}$ & 0.937 & 0.942 & 0.934 & 0.930 & 0.936 & 0.950 & 0.880 & 0.785 & 0.929 \\
\hline$H_{o}$ & $0.667 * *$ & $0.612 * *$ & $0.711 * *$ & $0.550 * *$ & $0.607 * *$ & $0.308 * *$ & $0.329 * *$ & $0.237 * *$ & $0.506 * *$ \\
\hline$F$ is & 0.290 & 0.353 & 0.241 & 0.412 & 0.353 & 0.679 & 0.628 & 0.701 & 0.455 \\
\hline \multicolumn{10}{|c|}{ CeATC1-22 } \\
\hline$N$ & 56 & 52 & 54 & 41 & 51 & 47 & 78 & 45 & 424 \\
\hline$N_{A}$ & $8(0)$ & $9(1)$ & $8(0)$ & $7(0)$ & $8(0)$ & $8(0)$ & $9(1)$ & $7(0)$ & 11 \\
\hline$R$ & $166-190$ & $163-187$ & $166-190$ & $166-184$ & $166-187$ & 169-190 & $135-190$ & $166-184$ & $135-190$ \\
\hline$R_{S}$ & 7.159 & 8.348 & 7.389 & 6.940 & 7.676 & 7.278 & 7.296 & 6.378 & 7.346 \\
\hline$A_{C}$ & 175 & 175 & 175 & 175 & $172 / 175$ & 175 & 175 & 175 & 175 \\
\hline$A_{C}$ freq & 0.429 & 0.298 & 0.370 & 0.329 & 0.265 & 0.0436 & 0.333 & 0.356 & \\
\hline$H_{e}$ & 0.742 & 0.821 & 0.764 & 0.787 & 0.812 & 0.739 & 0.780 & 0.781 & 0.781 \\
\hline$H_{o}$ & 0.750 & 0.885 & 0.704 & 0.756 & 0.902 & 0.787 & 0.872 & 0.889 & $0.821 * *$ \\
\hline$F$ is & -0.011 & -0.078 & 0.079 & 0.040 & -0.113 & -0.066 & -0.119 & -0.140 & -0.052 \\
\hline \multicolumn{10}{|c|}{ CeATC1-36 } \\
\hline$N$ & 63 & 51 & 49 & 37 & 52 & 46 & 77 & 43 & 418 \\
\hline$N_{A}$ & $5(0)$ & $5(0)$ & $6(0)$ & $5(0)$ & $7(0)$ & $6(0)$ & $6(0)$ & $6(0)$ & 9 \\
\hline$R$ & $135-57$ & $135-147$ & $132-157$ & $135-147$ & $129-147$ & $135-151$ & $135-157$ & $129-151$ & $129-157$ \\
\hline$R_{S}$ & 4.235 & 4.542 & 4.898 & 4.834 & 5.869 & 5.021 & 4.966 & 5.163 & 5.052 \\
\hline$A_{C}$ & 144 & 144 & 144 & 144 & 144 & 144 & 144 & 144 & 144 \\
\hline$A_{C}$ freq & 0.786 & 0.833 & 0.704 & 0.797 & 0.788 & 0.826 & 0.740 & 0.733 & \\
\hline$H_{e}$ & 0.367 & 0.299 & 0.473 & 0.355 & 0.369 & 0.311 & 0.428 & 0.441 & 0.384 \\
\hline$H_{o}$ & 0.333 & 0.255 & 0.367 & 0.378 & $0.308^{*}$ & 0.304 & 0.442 & 0.395 & $0.352 * *$ \\
\hline$F$ is & 0.091 & 0.149 & 0.225 & -0.066 & 0.167 & 0.020 & -0.032 & 0.105 & 0.085 \\
\hline \multicolumn{10}{|c|}{ CeATC1-52 } \\
\hline$N$ & 52 & 46 & 49 & 41 & 48 & 45 & 73 & 37 & 391 \\
\hline$N_{A}$ & $17(4)$ & $11(0)$ & $16(3)$ & $9(0)$ & $17(2)$ & $15(1)$ & $14(0)$ & $10(0)$ & 28 \\
\hline$R$ & $112-260$ & $123-181$ & $120-247$ & $130-181$ & $123-275$ & $109-181$ & $126-181$ & $109-181$ & $109-275$ \\
\hline$R_{S}$ & 13.602 & 10.191 & 13.655 & 8.673 & 14.563 & 12.406 & 9.983 & 9.326 & 11.822 \\
\hline$A_{C}$ & 136 & 136 & 136 & 136 & 136 & 136 & 136 & 139 & 136 \\
\hline$A_{C}$ freq & 0.346 & 0.348 & 0.276 & 0.463 & 0.354 & 0.433 & 0.342 & 0.257 & \\
\hline$H_{e}$ & 0.823 & 0.800 & 0.854 & 0.740 & 0.827 & 0.770 & 0.769 & 0.822 & 0.805 \\
\hline$H_{o}$ & 0.808 & 0.761 & 0.816 & 0.756 & 0.729 & 0.756 & $0.699 *$ & 0.838 & $0.765 * *$ \\
\hline$F$ is & 0.019 & 0.050 & 0.044 & -0.023 & 0.119 & 0.019 & 0.093 & -0.031 & 0.050 \\
\hline \multicolumn{10}{|c|}{ CeATC1-54 } \\
\hline$N$ & 61 & 53 & 57 & 41 & 60 & 47 & 72 & 45 & 436 \\
\hline$N_{A}$ & $18(1)$ & $17(1)$ & $17(2)$ & $13(1)$ & $18(0)$ & $19(1)$ & $21(4)$ & $15(3)$ & 40 \\
\hline$R$ & $141-215$ & 108-209 & $108-282$ & $108-185$ & 108-203 & $108-212$ & $108-282$ & $148-288$ & $108-288$ \\
\hline$R_{S}$ & 14.260 & 13.409 & 13.915 & 11.495 & 14.144 & 15.837 & 13.920 & 12.226 & 14.673 \\
\hline$A_{C}$ & 154 & 154 & 151 & 154 & 154 & 151 & 154 & 151 & 154 \\
\hline$A_{C}$ freq & 0.320 & 0.396 & 0.342 & 0.427 & 0.325 & 0.287 & 0.313 & 0.367 & \\
\hline$H_{e}$ & 0.811 & 0.771 & 0.818 & 0.759 & 0.835 & 0.836 & 0.799 & 0.749 & 0.804 \\
\hline
\end{tabular}


Table 1 continued

\begin{tabular}{|c|c|c|c|c|c|c|c|c|c|}
\hline & Ave & Sim & Boi & Cor & Fer & Bre & Oos & ILe & Total \\
\hline$H_{o}$ & $0.557 * *$ & $0.566^{*}$ & 0.684 & $0.537 *$ & $0.683 * *$ & $0.575^{* *}$ & $0.722 *$ & 0.533 & $0.617 * *$ \\
\hline$F$ is & 0.314 & 0.268 & 0.164 & 0.295 & 0.183 & 0.316 & 0.097 & 0.291 & 0.233 \\
\hline \multicolumn{10}{|c|}{ СeАTC2-4 } \\
\hline$N$ & 55 & 57 & 61 & 34 & 56 & 38 & 73 & 38 & 412 \\
\hline$N_{A}$ & $13(1)$ & $10(0)$ & $9(0)$ & $7(0)$ & $10(0)$ & $11(0)$ & $14(3)$ & $6(0)$ & 19 \\
\hline$R$ & $161-190$ & $155-190$ & $155-184$ & $161-178$ & $155-190$ & $155-255$ & $152-264$ & $164-255$ & $152-264$ \\
\hline$R_{S}$ & 10.205 & 8.253 & 7.251 & 6.817 & 8.630 & 10.346 & 10.878 & 5.968 & 10.206 \\
\hline$A_{C}$ & 172 & 172 & 172 & 172 & 172 & 172 & 172 & 175 & 172 \\
\hline$A_{C}$ freq & 0.518 & 0.526 & 0.623 & 0.559 & 0.625 & 0.382 & 0.377 & 0.474 & \\
\hline$H_{e}$ & 0.694 & 0.670 & 0.579 & 0.637 & 0.581 & 0.787 & 0.804 & 0.710 & 0.722 \\
\hline$H_{o}$ & $0.400 * *$ & $0.439 * *$ & $0.426^{*}$ & $0.412 * *$ & $0.464 *$ & 0.684 & $0.548 * *$ & $0.605^{*}$ & $0.490 * *$ \\
\hline$F$ is & 0.426 & 0.347 & 0.26 & 0.357 & 0.203 & 0.132 & 0.320 & 0.149 & 0.321 \\
\hline \multicolumn{10}{|c|}{ СеАТC2-11 } \\
\hline$N$ & 60 & 57 & 61 & 38 & 61 & 47 & 73 & 41 & 438 \\
\hline$N_{A}$ & $26(3)$ & $25(2)$ & $23(4)$ & $20(1)$ & $23(2)$ & $21(0)$ & $22(3)$ & $14(0)$ & 48 \\
\hline$R$ & $122-378$ & $122-372$ & $122-392$ & $122-335$ & $122-350$ & $122-378$ & $122-367$ & $122-274$ & $122-392$ \\
\hline$R_{S}$ & 18.279 & 18.584 & 17.708 & 18.361 & 17.159 & 17.486 & 16.435 & 12.980 & 17.659 \\
\hline$A_{C}$ & 214 & 214 & 214 & 214 & 170 & 170 & 170 & 170 & 170 \\
\hline$A_{C}$ freq & 0.217 & 0.175 & 0.221 & 0.197 & 0.189 & 0.245 & 0.240 & 0.232 & \\
\hline$H_{e}$ & 0.890 & 0.903 & 0.887 & 0.903 & 0.899 & 0.878 & 0.878 & 0.873 & 0.890 \\
\hline$H_{o}$ & $0.767 *$ & 0.842 & 0.853 & $0.763^{*}$ & 0.902 & $0.723 *$ & 0.808 & $0.561 * *$ & $0.790 * *$ \\
\hline$F$ is & 0.140 & 0.067 & 0.039 & 0.157 & -0.003 & 0.177 & 0.080 & 0.360 & 0.113 \\
\hline \multicolumn{10}{|c|}{ СеАТC2-12 } \\
\hline$N$ & 53 & 52 & 50 & 31 & 52 & 40 & 70 & 32 & 380 \\
\hline$N_{A}$ & $11(0)$ & $9(0)$ & $9(1)$ & $11(1)$ & $13(1)$ & $9(1)$ & $7(1)$ & $6(0)$ & 19 \\
\hline$R$ & $84-152$ & $84-152$ & $117-152$ & $84-167$ & $84-164$ & $84-164$ & $84-145$ & $84-142$ & $84-167$ \\
\hline$R_{S}$ & 9.534 & 7.782 & 8.033 & 11.000 & 11.229 & 8.218 & 5.550 & 5.968 & 9.287 \\
\hline$A_{C}$ & 123 & 123 & 129 & 123 & 123 & 123 & 123 & 123 & 123 \\
\hline$A_{C}$ freq & 0.396 & 0.481 & 0.400 & 0.387 & 0.462 & 0.538 & 0.407 & 0.391 & \\
\hline$H_{e}$ & 0.765 & 0.661 & 0.691 & 0.784 & 0.736 & 0.657 & 0.690 & 0.711 & 0.717 \\
\hline$H_{o}$ & $0.245^{* *}$ & $0.250 * *$ & $0.180 * *$ & $0.258 * *$ & $0.308^{* *}$ & $0.250 * *$ & $0.143 * *$ & $0.250 * *$ & $0.229 * *$ \\
\hline$F$ is & 0.682 & 0.624 & 0.742 & 0.674 & 0.584 & 0.623 & 0.794 & 0.652 & 0.681 \\
\hline \multicolumn{10}{|c|}{ СeATC2-34 } \\
\hline$N$ & 59 & 57 & 58 & 37 & 57 & 46 & 73 & 46 & 433 \\
\hline$N_{A}$ & $9(2)$ & $10(0)$ & $9(0)$ & $8(1)$ & $8(0)$ & $8(0)$ & $9(0)$ & $5(0)$ & 14 \\
\hline$R$ & $157-200$ & 154-194 & 157-194 & $157-197$ & 154-194 & $157-194$ & $157-194$ & $157-191$ & $154-200$ \\
\hline$R_{S}$ & 7.342 & 7.970 & 7.810 & 7.786 & 6.874 & 7.022 & 7.418 & 4.999 & 7.154 \\
\hline$A_{C}$ & 191 & 191 & 191 & 191 & 191 & 191 & 191 & 191 & 191 \\
\hline$A_{C}$ freq & 0.492 & 0.430 & 0.397 & 0.432 & 0.342 & 0.446 & 0.356 & 0.424 & \\
\hline$H_{e}$ & 0.697 & 0.751 & 0.769 & 0.747 & 0.763 & 0.735 & 0.774 & 0.731 & 0.750 \\
\hline$H_{o}$ & $0.661 *$ & 0.684 & 0.724 & 0.703 & 0.737 & 0.609 & 0.753 & 0.652 & $0.695 * *$ \\
\hline Fis & 0.052 & 0.090 & 0.059 & 0.060 & 0.034 & 0.173 & 0.027 & 0.109 & 0.074 \\
\hline \multicolumn{10}{|c|}{ CeATC2-44 } \\
\hline$N$ & 60 & 54 & 55 & 40 & 61 & 42 & 75 & 42 & 429 \\
\hline$N_{A}$ & $5(0)$ & $5(0)$ & $7(0)$ & $7(0)$ & $6(0)$ & $6(0)$ & $7(1)$ & $5(0)$ & 8 \\
\hline$R$ & $129-141$ & $129-141$ & $129-148$ & $129-148$ & $129-145$ & $129-145$ & $126-148$ & $129-141$ & $126-148$ \\
\hline$R_{S}$ & 4.285 & 4.499 & 5.933 & 6.492 & 5.815 & 5.926 & 6.236 & 4.672 & 5.604 \\
\hline$A_{C}$ & 129 & 129 & 135 & 135 & 135 & 129 & 129 & 135 & 135 \\
\hline
\end{tabular}


Table 1 continued

\begin{tabular}{|c|c|c|c|c|c|c|c|c|c|}
\hline & Ave & Sim & Boi & Cor & Fer & Bre & Oos & ILe & Total \\
\hline$A_{C}$ freq & 0.425 & 0.463 & 0.427 & 0.475 & 0.607 & 0.607 & 0.360 & 0.405 & \\
\hline$H_{e}$ & 0.633 & 0.610 & 0.669 & 0.643 & 0.582 & 0.587 & 0.720 & 0.684 & 0.665 \\
\hline$H_{o}$ & $0.633 * *$ & 0.519 & 0.709 & 0.625 & 0.557 & 0.500 & 0.653 & $0.405^{* *}$ & $0.585^{* *}$ \\
\hline$F$ is & -0.0007 & 0.151 & -0.060 & 0.028 & 0.042 & 0.150 & 0.094 & 0.411 & 0.120 \\
\hline \multicolumn{10}{|c|}{ СeATC2-51 } \\
\hline$N$ & 53 & 45 & 61 & 37 & 59 & 41 & 67 & 40 & 403 \\
\hline$N_{A}$ & $9(0)$ & $11(0)$ & $11(1)$ & $8(0)$ & $13(0)$ & $11(0)$ & $11(0)$ & $9(1)$ & 16 \\
\hline$R$ & $133-160$ & $133-164$ & $130-166$ & $137-160$ & $130-164$ & $133-160$ & $130-160$ & $130-169$ & $130-169$ \\
\hline$R_{S}$ & 8.217 & 10.765 & 9.163 & 7.785 & 11.653 & 10.270 & 9.232 & 8.492 & 9.913 \\
\hline$A_{C}$ & 151 & 147 & 151 & 151 & 151 & 151 & 151 & 137 & 151 \\
\hline$A_{C}$ freq & 0.283 & 0.289 & 0.303 & 0.432 & 0.305 & 0.293 & 0.306 & 0.300 & \\
\hline$H_{e}$ & 0.808 & 0.829 & 0.821 & 0.733 & 0.834 & 0.812 & 0.798 & 0.820 & 0.821 \\
\hline$H_{o}$ & $0.547 * *$ & $0.422 * *$ & $0.443 * *$ & $0.297 * *$ & $0.339 * *$ & $0.390 * *$ & $0.478 * *$ & 0.625 & $0.444 * *$ \\
\hline Fis & 0.325 & 0.493 & 0.463 & 0.598 & 0.596 & 0.522 & 0.404 & 0.240 & 0.459 \\
\hline \multicolumn{10}{|l|}{ All loci } \\
\hline$N$ & 63 & 60 & 64 & 41 & 62 & 48 & 78 & 46 & 462 \\
\hline$N_{A A}$ & 13 & 12.091 & 12.182 & 10.545 & 13.545 & 12.364 & 12.727 & 8.636 & 22.545 \\
\hline$R_{S A}$ & 10.6 & 10.312 & 10.297 & 9.948 & 11.36 & 10.981 & 9.915 & 7.958 & 10.758 \\
\hline$H_{e}$ & 0.742 & 0.732 & 0.751 & 0.729 & 0.743 & 0.733 & 0.756 & 0.736 & 0.752 \\
\hline$H_{o}$ & $0.579 * *$ & $0.567 * *$ & $0.602 * *$ & $0.549 * *$ & $0.594 * *$ & $0.535^{* *}$ & $0.586^{* *}$ & $0.545 * *$ & $0.572 * *$ \\
\hline$F$ is & 0.222 & 0.228 & 0.200 & 0.250 & 0.202 & 0.272 & 0.226 & 0.263 & 0.230 \\
\hline
\end{tabular}

$N$ number of individuals; $N_{\mathrm{A}}$ observed number of alleles (private alleles), $R$ size range of alleles in base pairs, $R_{\mathrm{S}}$ allelic richness based on a minimum sample size of 31 diploid individuals, $A_{\mathrm{C}}$ size in base pairs, $A_{\mathrm{C}}$ freq frequency of the most common allele, $H_{\mathrm{e}}$ expected heterozygosity, $H_{\mathrm{o}}$ observed heterozygosity, Fis inbreeding coefficient estimated following Weir and Cockerham (1984); $N_{A A}$ average allele number, $R_{S A}$ average allelic richness; * significant at $5 \%$ level; ** significant after sequential Bonferroni correction

Simón, Boiro, and Brest, while Ewens-Watterson-Slatkin exact test did so for the same locus-locality combinations and also for Oosterschelde at locus CeATC1-54 and for Isle of Lewis at loci CeATC2-34 and CeATC1-54 (data not shown).

Global multilocus $F$ st value was 0.015 , significantly different from zero $(P<0.001)$, and the standardized value was $F$ st' $^{\prime}=0.056$. Estimates of $F$ st per locus ranged from 0.001 (CeATC2-11) to 0.059 (CeATC2-4), three of them (CeATC1-52, CeATC2-4, and CeATC2-44) being significant at the $5 \%$ level (Table 2). These results fit in with those obtained when analyzing genic and genotypic differentiation using Genepop software, which detected significant differences $(P<0.05)$ for these three loci (data not shown). Of the 28 pair-wise $F$ st tests performed between localities, 20 were significant after Bonferroni correction (Table 3). Maximum differentiation was observed between Isle of Lewis and Ferrol $(F$ st $=0.049)$. Aveiro, San Simón, Boiro, and Corcubion localities were not significantly different from one another, while Ferrol was significantly different from all other localities except Corcubión. Brest, Oosterschelde, and Isle of Lewis were significantly differentiated from each other and from all other localities analyzed.
Table $2 F$ st values per locus and overall

\begin{tabular}{llc}
\hline Locus & $F$ st & $P$ value \\
\hline CeATC1-22 & 0.004 & 0.052 \\
CeATC1-36 & 0.004 & 0.145 \\
CeATC1-52 & 0.008 & $0.004^{*}$ \\
CeATC1-54 & 0.004 & 0.072 \\
CeATC2-4 & 0.059 & $<0.001^{*}$ \\
CeATC2-11 & 0.001 & 0.292 \\
CeATC2-34 & 0.004 & 0.069 \\
CeATC2-44 & 0.034 & $<0.001^{*}$ \\
Total & 0.015 & $<0.001^{*}$
\end{tabular}

* Significant at $5 \%$ level

Global analysis of molecular variance (AMOVA) detected a $1.55 \%$ variation among populations, while the percentage of variation within populations amounted to $98.44 \%$. Furthermore, based on the pair-wise $F$ st analysis results, a hierarchical analysis of molecular variance was carried out to test for the component of genetic diversity attributable to variance among different regional groups. When Aveiro, San Simón, Boiro, and Corcubión localities 
Table 3 Pair-wise Fst values (Weir and Cockerham 1984)

* Significant at $5 \%$ level; ** significant after sequential Bonferroni correction

\begin{tabular}{|c|c|c|c|c|c|c|c|}
\hline & Sim & Boi & Cor & Fer & Bre & Oos & ILe \\
\hline Ave & -0.001 & 0.000 & -0.002 & $0.008 * *$ & $0.007 *$ & $0.009 * *$ & $0.031 * *$ \\
\hline Sim & & $0.005^{*}$ & -0.003 & $0.008 * *$ & $0.010 * *$ & $0.012 * *$ & $0.034 * *$ \\
\hline Boi & & & 0.005 & $0.008 * *$ & $0.016^{* *}$ & $0.012 * *$ & $0.036 * *$ \\
\hline Cor & & & & 0.001 & $0.013 * *$ & $0.015 * *$ & $0.042 * *$ \\
\hline Fer & & & & & $0.036 * *$ & $0.019 * *$ & $0.049 * *$ \\
\hline Bre & & & & & & $0.013 * *$ & $0.035^{* *}$ \\
\hline Oos & & & & & & & $0.017 * *$ \\
\hline
\end{tabular}

Table 4 Analysis of molecular variance (AMOVA) performed for five groups inferred from pair-wise analysis; group 1 = Ferrol; group 2 = Corcubión, Boiro, San Simón, Aveiro; group 3 = Oosterschelde; group $4=$ Isle of Lewis; group $5=$ Brest

\begin{tabular}{lccc}
\hline Source of variation & Sum of squares & $\begin{array}{l}\text { Variance } \\
\text { components }\end{array}$ & $\begin{array}{l}\text { Percentage of } \\
\text { variation }\end{array}$ \\
\hline Among groups & 42.679 & 0.050 & 1.725 \\
Among populations within groups & 10.239 & 0.006 & 0.190 \\
Within populations & $2,398.546$ & 2.861 & 0.265 \\
Total & $2,451.464$ & 2.917 & 98.085 \\
\hline
\end{tabular}

Fixation indices: $F \mathrm{ct}=0.017, F \mathrm{sc}=0.002, F \mathrm{st}=0.019 ; *$ significant at $5 \%$ level

were grouped, AMOVA analysis identified an among groups significant component, explaining $1.73 \%$ of the total variance. Most of the variance was distributed within localities $(98.09 \%)$ and variance component among localities within groups was nonsignificant (Table 4). When Corcubión was excluded from this group and moved to form a group with Ferrol, variance component among groups kept significant $(P<0.001)$, explaining $1.59 \%$ of the total variance, but the variance component among localities within groups, although not significant, became slightly higher (data not shown).

Using Fst pair-wise estimates and based on combined data from the eight loci, Barrier software identified a barrier to gene flow in the English Channel (Fig. 1). Mantel test revealed a significant correlation between $F$ st and geographic distance $\left(R^{2}=0.441, P=0.005\right)$ when all eight localities were analyzed (Fig. 2).

\section{Discussion}

This study uses, for the first time in C. edule, microsatellite markers to analyze genetic variation and differentiation in samples collected along the European Atlantic coast. High levels of polymorphism and heterozygosity values were detected, with allele number per locus ranging from eight to 48 and global expected and observed heterozygosity values of 0.752 and 0.572 , respectively. This contrasts with values obtained for the same species using allozyme loci that showed lower number of alleles (8, Beaumont et al. 1980; 3-11, Beaumont and Pether 1996), and lower expected (0.28, Hummel et al. 1994; 0.322, Beaumont and Pether 1996) and observed (0.436, Beaumont et al. 1980; 0.24, Hummel et al. 1994; 0.278, Beaumont and Pether 1996) heterozygosity values. Thus, microsatellite markers were, as expected, more variable than allozyme markers.

All localities showed similar values of observed and expected heterozygosity, but significant differences between them were detected for allelic richness, with the lowest values obtained for localities sampled farther north; Isle of Lewis (7.958) and Oosterschelde (9.915), the latter

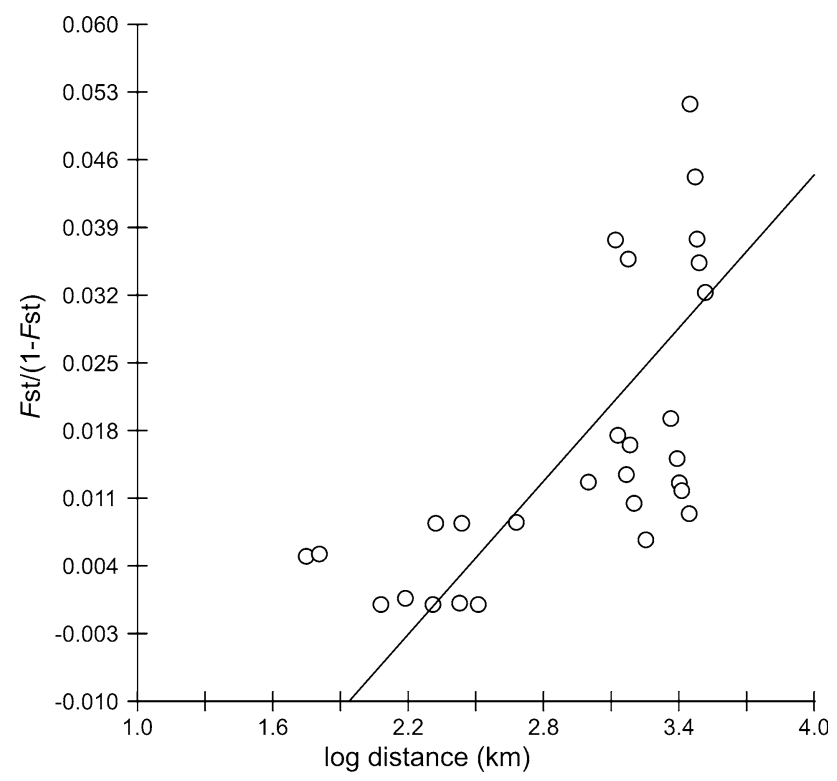

Fig. 2 Bivariate plot of log-transformed geographic distance against $F$ st $/(1-F$ st $)$ values 
being no significantly different from any other locality analyzed. This contrasts with what was detected by Krakau et al. (2012), who analyzed a fragment of the mitochondrial cytochrome c oxidase I gene and found higher genetic diversity values in samples of $C$. edule from the north of Europe than in those collected in the south. Indeed, within the southwestern group of their study, where almost all our localities are included, diversity values tend to increase as they move north. Even though our results do not agree with the existence of a northern refuge as postulated by the authors, differences might be caused by the use of nuclear markers against mitochondrial ones. Still, more sampling would be required to settle those differences.

However, our results are in line with the expectation that genetic diversity has an inverse relation with latitude due to the effect caused by Pleistocene glaciations, when marine fauna took shelter in the south (Hewitt 2004).

Compared to other bivalves, the mean number of alleles (22.545) in C. edule is in the range obtained with the same type of markers for oysters (18.533, Launey et al. 2002), clams (29.044, Vadopalas et al. 2004), and scallops (35.167, Kenchington et al. 2006). Overall expected and observed heterozygosity values obtained for $C$. edule were similar to those obtained for the other species of the genus (0.742 and 0.653 for C. glaucum, Tarnowska et al. 2010) but lower than those observed for other bivalve species (0.839 and 0.914, Launey et al. 2002; 0.937 and 0.596, Vadopalas et al. 2004; 0.797 and 0.731, Kenchington et al. 2006).

All but one locus-locality combinations that departed from HW expectations and all localities analyzed reported heterozygote deficits as showed by positive $F$ is values. Heterozygote deficits relative to HWE are common in marine bivalves and have been well documented not only for allozyme loci (Zouros and Foltz 1984; Gaffney 1990; Fairbrother and Beaumont 1993; Beaumont and Pether 1996) but for microsatellite markers as well (Launey et al. 2002; Hedgecock et al. 2004; Astanei et al. 2005; Carlsson and Reece 2007). Several biological and technical factors could account for the observed heterozygote deficit, such as inbreeding, Wahlund effect, selection and the presence of null alleles. The latter are the most likely cause for the heterozygote deficiencies detected here, as eight of the loci analyzed showed evidence for the presence of null alleles, three of them at frequencies above 0.2. Null alleles are a frequent feature in bivalves, a taxa often beset by amplification problems (Selkoe and Toonen 2006), and have been reported as the cause of heterozygosity deficit in many bivalve species such as geoduck clams (Vadopalas et al. 2004), oysters (Galindo-Sánchez et al. 2008), and mussels (Gardeström et al. 2008).

Although marine species' high potential for dispersal, along with the continuity of the marine habitat, tends to oppose isolation and divergence of populations, results obtained in this survey support the existence of genetic differentiation among $C$. edule localities along the European Atlantic coast. A significant genetic structure was revealed at the level of the whole study $(F \mathrm{st}=0.015$, $P<0.001)$. This $F$ st value is similar to those obtained in other studies that used microsatellite markers to assess genetic differentiation in other bivalve species such as oysters (Launey et al. 2002; Galindo-Sánchez et al. 2008). Morton et al. (1993) suggested that measures of differentiation for highly variable loci may be as much as an order of magnitude lower than that of traditional markers, such as allozymes. Therefore, estimates of differentiation were expected to be low because of the high variability of microsatellite loci, as the practical upper limit for $F$ st is actually the level of expected homozygosity, which is low for this type of markers, implying a reduction in their maximum $F$ st value (Charlesworth 1998; Hedrick 1999). Nevertheless, despite the effect of polymorphism on deflating $F$ st expectations, highly variable markers are valuable in exact tests of differentiation not only because of their sensitivity to gene flow (Ross et al. 1999) but also because their high variability gives them greater statistical power (Estoup et al. 1998). This has indeed been shown for several studies on aquatic species where microsatellites revealed a finer resolution of genetic structuring than allozymes (Ruzzante et al. 1999; Lundy et al. 2000; Lage et al. 2001; Mattiangeli et al. 2002; Knutsen et al. 2003; Taylor et al. 2003; Carlsson et al. 2004; Jorgensen et al. 2005). Considering the differences relative to $F$ st values depend on the marker used, a standardized value of $F$ st was computed $\left(F \mathrm{st}^{\prime}=0.056\right)$, indicating moderate genetic differentiation according to Wright's (1978) guidelines. This value is higher than those obtained with allozyme loci in the same species; Hummel et al. (1994) obtained an overall $F$ st value of 0.04 , detecting no significant differentiation between locations analyzed, while Beaumont and Pether (1996), with an average $F$ st of 0.015, detected little differentiation between samples coming from the UK southern coast.

$F$ st pair-wise values showed no differentiation between four localities situated northwest of the Iberian Peninsula (Aveiro, San Simón, Boiro, and Corcubión), which is supported by the AMOVA analysis, with a nonsignificant variance component among localities within groups that comprise $0.19 \%$ of the total variance (Table 4). This indicates the existence of high gene flow rates between them. Despite the distance between Aveiro and the Spanish localities, which doubles that between San Simón, Boiro, and Corcubión, all four localities were genetically similar. Aveiro is a shallow lagoon system with high tidal flow that allows a large water renovation. This may promote larval drift to the coastal waters (Joaquim et al. 2010) and be 
responsible for larvae dispersal, preventing genetic differentiation. Although Ferrol is also located northwest of the Iberian Peninsula, it showed significant differences to all other four Iberian localities analyzed but Corcubión. This may be due to their geographic position with respect to Cape Finisterre, where a major oceanographic boundary has been described (López-Jamar et al. 1992). Another possible explanation calls on the hypothesis of "Sweepstakes Reproductive Success", which suggests that the extremely large variance in individual reproductive success could produce, among other effects, the presence of genetic heterogeneity at reduced spatial scales (see review in Hedgecock and Pudovkin 2011).

Localities of Brest, Oosterschelde, and Isle of Lewis resulted in differences not only between them, but from all other localities analyzed. This could be a product of their geographical location, as gene flow modulates over greater distances and eventually reaches a level where the exchange of migrants is not strong enough to counter the effects of random genetic drift (Lind et al. 2007). The Mantel test revealed the existence of a significant correlation between pair-wise $F$ st estimates and geographical distance when all localities were analyzed. However, even though the analysis was statistically significant, graphic representation (Fig. 2) did not adjust well to a linear model; from a certain geographic distance onwards, genetic distance tends to increase sharply, more consistent with an exponential shape than a linear one. Therefore, samples at intermediate distances should be analyzed to confirm that genetic divergence between localities is a result of isolation by distance. It is important to note that geographical distance measures may not reflect real dispersal pathways, which are likely to be more complex. Isolation by distance may explain just part of the genetic differentiation observed. Nearshore circulation patterns may be affected by shoreline irregularities such as estuaries or bays, resulting in eddies and fronts that may retain larvae over time scales comparable with dispersal duration, and thus may account for enhanced local recruitment at specific locations (McShane et al. 1988; Dupont et al. 2007). This could be the case for the locality of Brest, where genetic isolation could also be influenced by the characteristics of the bay: a semi-enclosed marine ecosystem (Richard et al. 2006) favorable to the isolation of the larval pool and, in the long term, genetic isolation. Genetic isolation of this bay has already been observed for the molluscan species Crepidula fornicata (Dupont et al. 2007).

Barrier software suggested the presence of a barrier to gene flow situated in the English Channel. The precise situation of this barrier cannot be determined since no locations along the English Channel were analyzed. Other studies have found gene flow restrictions in this region, although the exact boundary location varied. Thus, Roman and Palumbi (2004) in their analysis of population structure in Carcinus maenas by means of mitochondrial cytochrome $\mathrm{c}$ oxidase I DNA found a significant break between western and northern Europe located between The Netherlands and Germany. In C. edule, the existence of such genetic discontinuity has also been described by Krakau et al. (2012) using a fragment of the same mitochondrial sequence. Other authors situate the genetic break further south; Jolly et al. (2005) found a phylogeographic break for the polychaete Pectinaria koreni that separates populations coming from Brittany and the English Channel. Also, Dupont et al. (2007) found a strong hierarchical structure for the molluscan species $C$. fornicata with two groups located on both sides of the Cotentin Peninsula. In spite of the still undefined location of the genetic barrier, it is clear that the English Channel currents play an important role in gene flow, with restrictions that may promote population differentiation.

Although microsatellite markers detected genetic differentiation among localities surveyed, the level of differentiation detected in $C$. edule is lower than in the other species of the genus, C. glaucum. In the latter, microsatellite markers detected a high level of genetic structure between localities along the coast of Europe (Tarnowska et al. 2010). Allozyme markers also showed a higher level of differentiation in C. glaucum with respect to C. edule (Hummel et al. 1994; Mariani et al. 2002; Nikula and Väinölä 2003). Hummel et al. (1994) pointed out that these differences could be explained by the different character of the species habitat, with $C$. glaucum individuals found in smaller, more or less limited water bodies, thus resulting in a more limited gene flow.

In conclusion, this work makes novel use of microsatellite markers to provide estimates of genetic diversity and population differentiation in $C$. edule from the Iberian Peninsula and other European locations. All localities displayed similar heterozygosity levels, but significant differences in allelic richness were observed, with the lowest values found in localities situated farther north. Localities from the Iberian Peninsula were genetically homogeneous, except for the one located farther north, and were different from those of other European sites, which may be partially explained by an isolation by distance process. Scottish, French, and Dutch localities displayed contrasting results, likely due to a combined effect of isolation by distance and the existence of barriers limiting gene flow between them. More localities should be analyzed by means of microsatellite markers in order to achieve complete knowledge of population structure and to establish the mechanisms that determine genetic differentiation in the cockle $C$. edule.

Acknowledgments We thank Jose García Gil and Rosa García Díaz for their technical assistance. This work was supported by Ministerio 
de Educación y Ciencia of the Spanish Government through project RZ2006-00005-00-00 and by Ministerio de Educación through a predoctoral FPU fellowship awarded to Luisa Martínez. We would also like to thank Dr. H. Hummel and Dr. O. van Hoesel for providing us with Oosterschelde cockle samples, and Dr. Rodrigo González Romero and Dr. Brad Williamson for their revision of English grammar and style.

\section{References}

Allendorf FW, England PR, Luikart G, Ritchie PA, Ryman N (2008) Genetic effects of harvest on wild animal populations. Trends Ecol Evol 23:327-337

Arias A, Fernández-Moreno M, Fernández-Tajes J, Gaspar MB, Méndez J (2011) Strong genetic differentiation among east Atlantic populations of the sword razor shell (Ensis siliqua) assessed with mtDNA and RAPD markers. Helgol Mar Res 65:81-89

Astanei I, Gosling E, Wilson J, Powell E (2005) Genetic variability and phylogeography of the invasive zebra mussel, Dreissena polymorpha (Pallas). Mol Ecol 14:1655-1666

Beaumont AR, Pether SMJ (1996) Allozyme variation and gene flow between cockle Cerastoderma edule populations in southern United Kingdom. Fish Res 28:263-275

Beaumont AR, Day TR, Gade G (1980) Genetic-variation at the octopine dehydrogenase locus in the adductor muscle of Cerastoderma edule (L.) and six other bivalve species. Mar Biol Lett 1:137-148

Belkhir K, Borsa P, Chikhi L, Raufaste N, Bonhomme F (2004) GENETIX 4.05, logiciel sous Windows TM pour la génétique des populations. Laboratoire Génome, Populations, Interactions, CNRS UMR 5171, Université de Montpellier II, Montpellier

Bortz J, Lienert G, Boehnke K (2000) Verteilungsfreie Methoden in der Biostatistik. Springer, Berlin

Brookfield JFY (1996) A simple new method for estimating null allele frequency from heterozygote deficiency. Mol Ecol 5:453-455

Carlsson J, Reece KS (2007) Eight PCR primers to amplify ESTlinked microsatellites in the eastern oyster, Crassostrea virginica genome. Mol Ecol Notes 7:257-259

Carlsson J, McDowell JR, Diaz-Jaimes P, Carlsson JEL, Boles SB, Gold JR, Graves JE (2004) Microsatellite and mitochondrial DNA analyses of Atlantic bluefin tuna (Thunnus thynnus thynnus) population structure in the Mediterranean Sea. Mol Ecol 13:3345-3356

Chapuis MP, Estoup A (2007) Microsatellite null alleles and estimation of population differentiation. Mol Biol Evol 24:621-631

Charlesworth B (1998) Measures of divergence between populations and the effect of forces that reduce variability. Mol Biol Evol 15:538-543

Dare PJ, Bell MC, Walker P, Bannister RCA (2004) Historical and current status of cockle and mussel stocks in The Wash. CEFAS, Lowestoft

Dupont L, Ellien C, Viard F (2007) Limits to gene flow in the slipper limpet Crepidula fornicata as revealed by microsatellite data and a larval dispersal model. Mar Ecol Prog Ser 349:125-138

Estoup A, Rousset F, Michalakis Y, Cornuet JM, Adriamanga M, Guyomard R (1998) Comparative analysis of microsatellite and allozyme markers: a case study investigating microgeographic differentiation in brown trout (Salmo trutta). Mol Ecol 7:339-353

Excoffier L, Laval G, Schneider S (2005) Arlequin (version 3.0): an integrated software package for population genetics data analysis. Evol Bioinform Online 1:47-50
Fairbrother JE, Beaumont AR (1993) Heterozygote deficiencies in a cohort of newly settled Mytilus edulis spat. J Mar Biol Assoc UK 73:647-653

Fernández-Tajes J, Méndez J (2007) Identification of the razor clam species Ensis arcuatus, E. siliqua, E. directus, E. macha, and Solen marginatus using PCR-RFLP analysis of the 5S rDNA region. J Agric Food Chem 55:7278-7282

Gaffney PM (1990) Enzyme heterozygosity, growth-rate, and viability in Mytilus edulis - another look. Evolution 44:204-210

Galindo-Sánchez CE, Gaffney PM, Pérez-Rostro CI, De La RosaVélez J, Candela J, Cruz P (2008) Assessment of genetic diversity of the eastern oyster Crassostrea virginica in Veracruz, Mexico using microsatellite markers. J Shellfish Res 27:721-727

Gardeström J, Pereyra RT, Andre C (2008) Characterization of six microsatellite loci in the Baltic blue mussel Mytilus trossulus and cross-species amplification in North Sea Mytilus edulis. Conserv Genet 9:1003-1005

Goudet J (2002) FSTAT, a program to estimate and test gene diversities and fixation indices (version 2.9.3.2). http://www. unil.ch/izea/softwares/fstat.html

Hayward PJ, Ryland JS (1995) Handbook of the marine fauna of north-west Europe. Oxford University Press, Oxford

Hedgecock D, Pudovkin AI (2011) Sweeptakes reproductive success in highly fecund marine fish and shellfish: a review and commentary. B Mar Sci 84:971-1002

Hedgecock D, Li G, Hubert S, Bucklin K, Ribes V (2004) Widespread null alleles and poor cross-species amplification of microsatellite DNA loci cloned from the Pacific oyster, Crassostrea gigas. J Shellfish Res 23:379-385

Hedrick PW (1999) Perspective: highly variable loci and their interpretation in evolution and conservation. Evolution 53:313-318

Hedrick PW (2005) A standardized genetic differentiation measure. Evolution 59:1633-1638

Hewitt GM (2004) Genetic consequences of climatic oscillations in the quaternary. Philos Trans R Soc of Lond B Biol Sci 359:183-195

Honkoop PJC, van der Meer J (1998) Experimentally induced effects of water temperature and immersion time on reproductive output of bivalves in the Wadden Sea. J Exp Mar Biol Ecol 220:227-246

Hummel H, Wolowicz M, Bogaards RH (1994) Genetic-variability and relationships for populations of Cerastoderma edule and of the C. glaucum complex. Neth J Sea Res 33:81-89

Jensen JL, Bohonak AJ, Kelley ST (2005) Isolation by distance, web service. BMC Genet 6:13

Joaquim S, Pereira J, Leitao A, Matias D, Chaves R, Guedes-Pinto H, Chícharo L, Gaspar M (2010) Genetic diversity of two Portuguese populations of the pullet carpet shell Venerupis senegalensis, based on RAPD markers: contribution to a sustainable restocking program. Helgol Mar Res 64:289-295

Jolly MT, Jollivet D, Gentil F, Thiebaut E, Viard F (2005) Sharp genetic break between Atlantic and English Channel populations of the polychaete Pectinaria koreni, along the North coast of France. Heredity 94:23-32

Jorgensen HBH, Hansen MM, Bekkevold D, Ruzzante DE, Loeschcke V (2005) Marine landscapes and population genetic structure of herring (Clupea harengus L.) in the Baltic Sea. Mol Ecol 14:3219-3234

Kenchington EL, Patwary MU, Zouros E, Bird CJ (2006) Genetic differentiation in relation to marine landscape in a broadcastspawning bivalve mollusc (Placopecten magellanicus). Mol Ecol 15:1781-1796

Knutsen H, Jorde PE, Andre C, Stenseth NC (2003) Fine-scaled geographical population structuring in a highly mobile marine species: the Atlantic cod. Mol Ecol 12:385-394 
Krakau M, Jacobsen S, Jensen KT, Reise K (2012) The cockle Cerastoderma edule at Northeast Atlantic shores: genetic signatures of glacial refugia. Mar Biol 159:221-230

Lage C, Purcell M, Fogarty M, Kornfield I (2001) Microsatellite evaluation of haddock (Melanogrammus aeglefinus) stocks in the northwest Atlantic Ocean. Can J Fish Aquat Sci 58:982-990

Launey S, Ledu C, Boudry P, Bonhomme F, Naciri-Graven Y (2002) Geographic structure in the European flat oyster (Ostrea edulis L.) as revealed by microsatellite polymorphism. J Hered 93:331-338

Lind CE, Evans BS, Taylor JJU, Jerry DR (2007) Population genetics of a marine bivalve, Pinctada maxima, throughout the IndoAustralian Archipelago shows differentiation and decreased diversity at range limits. Mol Ecol 16:5193-5203

López-Jamar E, Cal RM, González G, Hanson RB, Rey J, Santiago G, Tenore KR (1992) Upwelling and out welling effects on the benthic regime of the continental shelf off Galicia, NW Spain. J Mar Res 50:465-488

Lundy CJ, Rico C, Hewitt GM (2000) Temporal and spatial genetic variation in spawning grounds of European hake (Merluccius merluccius) in the Bay of Biscay. Mol Ecol 9:2067-2079

Luttikhuizen PC, Drent J, van Delden W, Piersma T (2003) Spatially structured genetic variation in a broadcast spawning bivalve: quantitative vs. Molecular traits. J Evol Biol 16:260-272

Manni F, Guérard E, Heyer E (2004) Geographic patterns of (genetic, morphologic, linguistic) variation: how barriers can be detected by "Monmonier's algorithm". Hum Biol 76:173-190

Mariani S, Ketmaier V, de Matthaeis E (2002) Genetic structuring and gene flow in Cerastoderma glaucum (Bivalvia: Cardiidae): evidence from allozyme variation at different geographic scales. Mar Biol 140:687-697

Martínez L, Arias A, Méndez J, Insua A, Freire R (2009) Development of twelve polymorphic microsatellite markers in the edible cockle Cerastoderma edule (bivalvia: Cardiidae). Conserv Genet Resour 1:107-109

Mattiangeli V, Galvin P, Ryan AW, Mork J, Cross TF (2002) VNTR variability in Atlantic poor cod (Trisopterus minutus minutus) throughout its range: single locus minisatellite data suggest reproductive isolation for the Faroe Bank population. Fish Res 58:185-191

McShane PE, Black KP, Smith MG (1988) Recruitment processes in Haliotis rubra (Mollusca, Gastropoda) and regional hydrodynamics in south eastern Australia imply localized dispersal of larvae. J Exp Mar Biol Ecol 124:175-203

Morton NE, Collins A, Balazs I (1993) Kinship bioassay on hypervariable loci in blacks and caucasians. Proc Natl Acad Sci USA 90:1892-1896

Nei M (1978) Estimation of average heterozygosity and genetic distance from a small number of individuals. Genetics 89:583-590

Nikula R, Väinölä R (2003) Phylogeography of Cerastoderma glaucum (Bivalvia: Cardiidae) across Europe: a major break in the Eastern Mediterranean. Mar Biol 143:339-350

Reeb CA, Avise JC (1990) A genetic discontinuity in a continuously distributed species - mitochondrial-DNA in the American oyster, Crassostrea virginica. Genetics 124:397-406
Rice WR (1989) Analyzing tables of statistical tests. Evolution 43:223-225

Richard J, Huet M, Thouzeau G, Paulet YM (2006) Reproduction of the invasive slipper limpet, Crepidula fornicata, in the Bay of Brest, France. Mar Biol 149:789-801

Ridgway G (2001) Interpopulation variation in blue mussels, Mytilus edulis L., over short distances. Sarsia 86:157-161

Roman J, Palumbi SR (2004) A global invader at home: population structure of the green crab, Carcinus maenas, in Europe. Mol Ecol 13:2891-2898

Ross KG, Shoemaker DD, Krieger MJB, DeHeer CJ, Keller L (1999) Assessing genetic structure with multiple classes of molecular markers: a case study involving the introduced fire ant Solenopsis invicta. Mol Biol Evol 16:525-543

Rousset F (2008) Genepop'007: a complete reimplementation of the Genepop software for Windows and Linux. Mol Ecol Notes 8:103-106

Ruzzante DE, Taggart CT, Cook D (1999) A review of the evidence for genetic structure of cod (Gadus morhua) populations in the NW Atlantic and population affinities of larval cod off Newfoundland and the Gulf of St. Lawrence. Fish Res 43:79-97

Selkoe KA, Toonen RJ (2006) Microsatellites for ecologists: a practical guide to using and evaluating microsatellite markers. Ecol Lett 9:615-629

Slatkin M (1994) An exact test for neutrality based on the Ewens sampling distribution. Genet Res 64:71-74

Slatkin M (1996) A correction to the exact test based on the Ewens sampling distribution. Genet Res 68:259-260

Tarnowska K, Chenuil A, Nikula R, Féral JP, Wolowicz M (2010) Complex genetic population structure of the bivalve Cerastoderma glaucum in a highly fragmented lagoon habitat. Mar Ecol Prog Ser 406:173-184

Taylor EB, Stamford MD, Baxter JS (2003) Population subdivision in westslope cutthroat trout (Oncorhynchus clarki lewisi) at the northern periphery of its range: evolutionary inferences and conservation implications. Mol Ecol 12:2609-2622

Vadopalas B, Leclair LL, Bentzen P (2004) Microsatellite and allozyme analyses reveal few genetic differences among spatially distinct aggregations of geoduck clams (Panopea abrupta, Conrad 1849). J Shellfish Res 23:693-706

Van Oosterhout C, Hutchinson WF, Wills DPM, Shipley P (2004) Micro-Checker: software for identifying and correcting genotyping errors in microsatellite data. Mol Ecol Notes 4:535-538

Watterson GA (1978) Homozygosity test of neutrality. Genetics 88:405-417

Watterson GA (1986) The homozygosity test after a change in population-size. Genetics 112:899-907

Weir BS, Cockerham CC (1984) Estimating F-statistics for the analysis of population-structure. Evolution 38:1358-1370

Wright S (1978) Evolution and the genetics of populations. variability within and among natural populations, vol 4. University of Chicago Press, Chicago

Zouros E, Foltz DW (1984) Possible explanations of heterozygote deficiency in bivalve mollusks. Malacologia 25:583-591 